\title{
A Review of Dementia with Lewy Bodies' Impact, Diagnostic Criteria and Treatment
}

\author{
Samuel D. Capouch · Martin R. Farlow · Jared R. Brosch
}

Received: May 23, 2018 / Published online: July 9, 2018

(C) The Author(s) 2018

\section{ABSTRACT}

Dementia with Lewy bodies is one of the most common causes of dementia. It is not as common as Alzheimer's disease; the general public's awareness of the disease is poor in comparison. Its effects on caregivers and patients alike are not well known to the general population. There are currently no FDA-approved medications specifically for the treatment of DLB. Many of the medications that are approved for Alzheimer's disease are widely used in the treatment of DLB with varying degrees of success. Treatment of DLB is life long and requires a dedicated team of physicians and caregivers to minimize the degree of morbidity and mortality experienced by the patients suffering from the disease as it progresses.

Keywords: Alpha-synuclein; Dementia; Dementia with Lewy bodies; Lewy body dementia

Enhanced digital content To view enhanced digital content for this article go to https://doi.org/10.6084/ m9.figshare.6703613.

S. D. Capouch · M. R. Farlow · J. R. Brosch $(\bowtie)$ Department of Neurology, Indiana University School of Medicine, 355 W 16th St \#4700, Indianapolis, IN 46202, USA

e-mail: jbrosch@iupui.edu

\section{INTRODUCTION}

Dementia is defined as measurable cognitive impairment in conjunction with an impact on activities of daily living. It encompasses a broad constellation of conditions including neurodegenerative disease. Dementia often includes difficulty with language, memory, impulsivity, and communication and additionally can lead to changes in behavior and mood. Dementia with Lewy bodies is one of several neurodegenerative diseases that can lead to dementia and its associated symptoms in patients. Patients who are diagnosed with dementia with Lewy bodies often have no family history of the disease. The disease is caused by an accumulation of alpha-synuclein protein within neurons in the brain; these protein aggregates are known as Lewy bodies [1].

\section{Incidence/Prevalence}

In 2002, it was estimated that 3.4 million people in the USA over the age of 71 years had dementia of some kind. Increasing age is the most significant risk factor for developing dementia; prevalence increases from $5.0 \%$ in the age range of $71-79$ to $37.4 \%$ in those aged 90 or older [2]. Alzheimer's disease is the most common cause of dementia in the USA. Dementia with Lewy bodies and vascular dementia are widely considered to be the most prevalent causes of dementia after Alzheimer's 
[2-4]. As the proportion of the population that is elderly increases, proper prevention and treatment of dementing conditions are imperative because of the wide-ranging implications for patients and caregivers alike. The incidence of DLB is strongly associated with increasing age and male gender. The difference in incidence between men and women is not currently explained but may be due to endocrine, genetic, environmental, and other factors [5]. Historically, the diagnosis of DLB has been confounded because of lack of consensus on diagnostic criteria as well as its similarities to other dementing conditions. Thanks to new criteria that aid identification, the incidence of DLB has been found to account for approximately 1 out of 25 new diagnoses of dementia in the general population. In secondary care, DLB accounts for 1 out of 13 new diagnoses of dementia-this is likely due to greater awareness of diagnostic criteria among specialists [3].

\section{Morbidity/Mortality}

Dementia with Lewy bodies has significant implications for the health and well-being of patients. Outcomes in patients who suffer from DLB are poor, particularly when contrasted with patients who have AD. Patients with DLB have more neuropsychiatric features at initial presentation as well as follow-up than patients with AD. The risk of hospitalization or death due to adverse events such as falls and respiratory infections is greater in DLB than AD. Despite the differences in the frequency of adverse events, reviews have shown there is little difference in the rate of decline of cognitive and neuropsychiatric function among vascular dementia, AD, and DLB $[6,7]$. Median age at death is similar between AD and DLB; however, the time from diagnosis to death is shorter in $\mathrm{DLB}$ than $\mathrm{AD}$. This may be partially explained by a delay in diagnosis of DLB. This exemplifies the importance of refined and accurate diagnostic criteria for DLB. Survival time from presentation of symptoms as well as from presentation to psychiatric services is similar between DLB and AD patients [8]. The environment has been observed to have an effect on the mortality of patients who suffer from DLB. Mortality risk in DLB patients is particularly increased compared with Alzheimer's disease patients in a nursing home setting [9]. Patients with DLB have limited mobility and rely heavily upon caregivers to prevent falls and other injuries. Without active caregivers, complication rates in DLB patients are high. Additional factors that can expedite mortality in DLB include: neuroleptic sensitivity, potentially higher rates of hospitalization due to delirium/ encephalopathy, and higher rates of orthostatic hypotension/autonomic dysfunction.

\section{Impact}

The impact of dementia is not limited to the patients. The financial impact of DLB on patients and caregivers tends to increase as the disease progresses [10]. Besides financial concerns, caregivers have reported on surveys that they have fears of the future excessive stress, loss of a social life, and uncertainty about what to do next. Many caregivers report feelings of isolation, and no one can understand the responsibility that they have. A valuable tool that many caregivers of patients with dementia utilize is respite care or hired inhome assistance. However, only a minority of caregivers have been found to utilize these services to help them care for their loved ones who have DLB [11]. Patients who suffer from DLB have loss of independence early on in the course of their disease, including reliance on caregivers to complete activities of daily living [12]. Quality of life is adversely affected in DLB: compared with their peers who suffer from Alzheimer's disease, DLB patients have been found to have greatly reduced quality of life measurements on assessment regardless of whether or not quality-of-life assessments were performed by caregivers or patients. Delusions and apathy, common in patients with DLB, are strongly correlated with diminished quality of life [13]. Establishing a diagnosis for DLB can be difficult, despite the advent of new diagnostic criteria. The diagnostic 
process of DLB is often frustrating for caregivers and patients alike. Half of the caregivers of patients from a web-based survey reported seeing over three doctors for more than ten visits before the diagnosis of DLB was established. While neurologists were reportedly responsible for $62 \%$ of these diagnoses, primary care physicians were only responsible for $6 \%$. This provides evidence for a disproportionate misunderstanding of the diagnostic criteria, or lack of recognition, among primary care physicians compared with specialists [14]. The importance of making a correct diagnosis in degenerative dementia is crucial to patient outcomes. Objective measurements of disease burden show that diagnosis of DLB leads to a three-fold increase in resource utilization in addition to the significantly lower reported quality of life compared with a diagnosis of Alzheimer's disease [13].

The aim of the current study is to provide an overview of the current diagnosis, treatment, and investigational work being conducted in patients with DLB. This article is based on previously conducted studies and does not contain any studies with human participants or animals performed by any of the authors.

\section{RECOGNITION/DIAGNOSIS}

\section{Neuropathology}

The clinical symptoms of dementia with Lewy bodies can often overlap with those of Alzheimer's and Parkinson's diseases. It is important to note that mixed pathology cases commonly occur; in certain studies distinguishing features of both DLB and AD have been seen in up to half of research patients at autopsy [15]. Lewy bodies, the hallmark brain histopathology of DLB, are not specific to DLB. These protein aggregates are also found in other diseases including multiple systems atrophy and Parkinson's disease. A key histopathologic difference in these conditions is the distribution of these alpha-synuclein aggregates. In Parkinson's disease, they are found primarily within the dopaminergic neurons of the substantia nigra within the brainstem. In DLB, the Lewy bodies are diffusely distributed throughout the cortices of the brain [16]. Another key difference in neuropathology between Parkinson's and DLB is the microscopic appearance of these Lewy bodies: the cortically distributed Lewy bodies of DLB are less eosinophilic, less distinctly demarcated, and less filamentous than those of the Lewy bodies of the brainstem in PD [17]. Lewy bodies have been found in familial AD, AD associated with Down's syndrome, and sporadic AD, most commonly restricted to the amygdala [18]. There is some thought that, due to the heterogeneous nature of the deficiencies in neurotransmitters associated with Alzheimer's disease, DLB and other neurodegenerative diseases, they represent a spectrum of disease rather than solitary and distinct conditions. Skeptics argue that, due to the varying presentation of Lewy body disease among affected patients, it is possible that DLB is just a subpopulation suffering from an already known and recognized disease possessing uniquely differentiating characteristics (e.g., neuroleptic sensitivity) [19]. While it is important to consider similarities between the various neurodegenerative disorders and the likelihood of mixed pathology cases, current understanding of the available literature requires clinicians to utilize a similar but individually tailored approach to treatment of patients with these different diagnoses. Finally, this heterogeneous pathologic milieu may also have implications for the targeted approach of current experimental treatments of these conditions and likely implies a need for in vivo characterization and tailored multifaceted approaches.

\section{Clinical Features}

Lewy body dementia is an umbrella term that encompasses both Parkinson's disease dementia and DLB. In a clinical setting, it may be difficult to distinguish between DLB and Parkinson's disease dementia if the temporal relationship between symptoms has not been well documented. Generally the onset of dementia and parkinsonism occurs concurrently in DLB. In research, applying a 1-year rule between the onset of parkinsonism and dementia can help distinguish between DLB and Parkinson's 
disease dementia [20]. In contrast, the mean duration of Parkinson's symptoms prior to dementia in Parkinson's disease dementia is 10 years [21]. The Consortium on Dementia with Lewy Bodies recommendations and consensus criteria have recently been revised and improved. These changes were put in place with the goal of increased sensitivity in the detection of the disease in the hopes of improving on what was previously seen as unsatisfactory accurate diagnosing of patients suffering from DLB $[3,20]$. Diagnosis of DLB depends on the presence of core clinical features in the setting of a progressive dementia, which often has cognitive deficits in attention, executive functioning, and visuospatial abilities. The core clinical features, supportive clinical features, core (indicative) biomarkers, and supportive biomarkers are included in Table 1. Diagnosis of DLB can be further differentiated between "probable" and "possible" diagnosis. "Probable" DLB can be diagnosed if (in addition to dementia) either two of the core clinical features are present with or without the presence of indicative biomarkers or only one core clinical feature is present alongside one or more of the indicative biomarkers. "Possible" DLB can be diagnosed if only one core clinical feature of DLB is present with no indicative biomarkers or if one or more of the indicative biomarkers are present but there are no core clinical features [20].

Dementia is the essential hallmark symptom of DLB. Dementia by definition must be severe enough to interfere with a patient's day-to-day functioning, often interfering with occupational and social activities. Screening tests such as the Mini-Mental State Examination (MMSE) and Montreal Cognitive Assessment (MOCA) have utility in screening a patient's overall mentation and impairment. These tests alone may not be adequate in establishing a conclusive neurocognitive assessment in DLB patients. A thorough assessment for DLB involves in depth testing of the most commonly affected domains of cognition including visual processing, attention, and executive function. In contrast to early memory impairment of $\mathrm{AD}$, memory impairment of DLB is not an early prominent feature, instead appearing later in the course of the disease [17]. Comparisons of cognition between DLB versus $\mathrm{AD}$ and DLB versus normal controls have shown significant deficits in attention/concentration, visual perception/construction, naming, letter fluency, and verbal memory in the DLB groups. In distinguishing between patients with DLB and AD, particular attention should be paid to trailmaking tests and complex figure copying. Both $\mathrm{AD}$ and $\mathrm{DLB}$ patients have impairments in attention and visual perception/construction tests compared with normal controls. However, DLB patients have impairments to a much greater extent [22]. Fluctuations in cognition are a core clinical feature of DLB. These fluctuations are most useful in distinguishing DLB early in the course of the disease. The qualitative features of the cognition fluctuations in DLB are generally regarded as their distinguishing feature compared with the cognitive fluctuations of other dementing conditions. Studies that assess caregiver perceptions of cognition fluctuations provide descriptions such as episodes of "blank staring" or disengagement in DLB in contrast to the episodes of confusion (such as repetition of conversation or task-based/situational confusion) common to AD. Episodic confusion in DLB patients has been observed to have confabulatory or delusional qualities as well. Additionally, daytime somnolence is reported more frequently among caregivers of DLB patients. Cognitive fluctuations regardless of description have been shown to be more frequent in DLB patients than in those with $\mathrm{AD}$ [23].

Visual hallucinations are prevalent in patients who suffer from DLB, occurring in up to $80 \%$ of patients. These hallucinations are often described as people, children, or animals. Other visual perceptive symptoms such as flashing lights, visual patterns, and shadows have been reported. Patients may respond to these hallucinations with varying degrees of insight and emotional reaction [20]. Visual hallucinations at initial presentation have a very strong positive predictive value for a diagnosis of dementia with Lewy bodies (and lack of visuospatial impairment being the best negative predictor for the disease) [24]. The presence of early, severe visuospatial deficits is a good 
Table 1 Diagnostic criteria for dementia with Lewy bodies Adapted from McKeith et al. [20]

\begin{tabular}{ll}
\hline Essential feature & $\begin{array}{l}\text { Diagnosis of dementia with progressive decline and often with features of impaired } \\
\text { executive functioning, visuospatial abilities, and attention }\end{array}$ \\
\hline Core clinical features & Fluctuations in cognition with pronounced changes in attention/alertness \\
& Recurrent visual hallucinations \\
& REM behavior disorder \\
& One or more spontaneous cardinal features of parkinsonism \\
Supportive clinical & Neuroleptic sensitivity \\
features & Postural instability \\
& Repeated falls \\
& Syncope or episodes of unresponsiveness \\
& Autonomic dysfunction \\
& Hypersomnia \\
& Hyposmia \\
& Hallucination in other modalities \\
& Delusional thoughts \\
& Apathy \\
Anxiety/depression & Reduced dopamine transporter uptake on SPECT/PET \\
Low uptake on ${ }^{123}$ iodine-MIBG myocardial scintigraphy \\
Polysomnographic evidence of REM sleep without atonia \\
MRI with relative preservation of mesial temporal lobe structures \\
SPECT/PET with generalized low uptake and reduced occipital activity \\
EEG with prominent slow wave activity and fluctuations into pre-alpha/theta range \\
\hline indicative) & \\
&
\end{tabular}

indicator for the development of visual hallucinations. DLB patients who have severe visuospatial deficits early on in the course of their disease are more likely to develop these visual hallucinations than patients with more mild deficits [25]. Clinical observation or reporting on thorough history taking of visual hallucinations and generalized visuospatial impairments are very important for the differentiation between DLB and AD. Like visual hallucinations, auditory and tactile hallucinations have been reported in patients with DLB to varying degrees, although to a lesser extent [26]. These hallucinations can occasionally lead to episodes of disabling psychosis; however, caregivers should exercise caution in the use of neuroleptic medications.

Neuroleptic sensitivity is a widely recognized feature of DLB. Reactions to neuroleptics in these patients can include paradoxical agitation, increases in parkinsonian motor and extrapyramidal symptoms, and neuroleptic malignant syndrome. Severe neuroleptic sensitivity in DLB patients has been observed to be associated with increased mortality and markedly decreased survival time from onset of 
psychiatric symptoms compared with other types of dementia [27]. Treatment with first generation neuroleptics portends higher risk of reaction than atypical (second generation) neuroleptics. Low doses of atypical neuroleptics may sometimes be used in treatment if hallucinations are having a major impact on quality of life.

Parkinsonian motor symptoms are common in DLB, with up to $85 \%$ of patients experiencing them [20]. Motor symptoms of DLB patients are similar to PD patients. These symptoms primarily include rest tremor, bradykinesia, muscular rigidity, and axial instability/gait disturbance. In the authors' clinical experience, rest tremor is less prevalent in DLB than in idiopathic Parkinson's disease, and this is also supported by some limited published data [28]. Another study showed more bradykinesia, more severe action tremor, and more rigidity than in idiopathic Parkinson's patients [29]. This implies that factors other than nigral cell loss may contribute to EPS symptoms in DLB patients.

REM sleep behavior disorder is a commonly reported core clinical feature of DLB. REM sleep behavior can precede DLB by many years and, in some cases, even decades [30-32]. Among REM sleep behavior disorder patients, cognitive tests that evaluate executive functions and attention have been shown to reliably predict eventual development of dementia [33]. Patients with DLB who have probable REM sleep behavior disorder also have been found to have earlier and more rapid onset of the core clinical features of DLB, including parkinsonism and hallucinations [34]. Polysomnography may be helpful to definitively diagnose REM sleep behavior disorder and rule out other etiologies of sleep disturbance among DLB patients and is one of the core (indicative) biomarkers.

Dysautonomia, while not generally considered a core clinical feature of dementia with Lewy bodies, is a supportive feature in the diagnosis of the disease. Symptoms of autonomic dysfunction include orthostatic hypotension, tachycardia, incontinence, and constipation [20]. This dysautonomia in patients with DLB is associated with shorter survival. The symptom of dysautonomia that is most closely correlated to decreased survival is orthostatic hypotension; the symptoms of constipation and incontinence are obviously disabling, but are not particularly specific to autonomic dysfunction as they are common in the elderly populations [35]. Delusional thoughts can occur in DLB with the most common delusion being Capgras syndrome. In this syndrome, the patient believes that his loved one is an "imposter." Clinically, patients will often question their spouse and ask where their spouse is. They will ask "are we married?" They may recognize that there are multiple copies of their spouse and can either name or designate which version of their spouse is present. In the clinical experience of the authors, Capgras is the most common delusion, and it is more prevalent in DLB than in other types of dementia.

\section{Biomarkers/Imaging}

Biomarkers can be helpful in the diagnostic process of DLB. The diagnosis of probable DLB can be made if one or more of the indicative biomarkers is associated with one or more of the aforementioned core clinical features. If dementia is present in the absence of core clinical features but with one or more of the indicative biomarkers, probable DLB is instead diagnosed [20]. Low dopamine transporter reuptake in the basal ganglia shown by SPECT or PET imaging is a now widely accepted indicative biomarker for probable DLB. Sensitivity for DLB diagnosis with one of these scans has been shown to be $78 \%$, with a specificity of 90\% for excluding non-DLB dementia (primarily AD). This can be helpful in differentiation between DLB and AD [36]. Reduced uptake of ${ }^{123}$ iodine-MIBG on myocardial scintigraphy is another widely accepted indicative biomarker for DLB. Further decreased uptake of ${ }^{123}$ iodineMIBG has been shown to correlate with increased severity of cognitive impairments between patients with severe impairments compared with those with mild or moderate impairments, as well as between patients with and without orthostatic hypotension [37]. ${ }^{123} \mathrm{I}$ MIBG myocardial scintigraphy has also been 
shown to reliably predict conversion to probably DLB from possible DLB [38]. ${ }^{123}$ I-MIBG myocardial scintigraphy is more widely available in Europe and less available in the US. A third indicative biomarker for DLB is the presence of distinctive polysomnographic findings showing REM sleep that occurs without atonia. One retrospective study over an 11-year period of DLB patients who underwent PSG showed 96\% experienced REM sleep without atonia [39].

Supportive biomarkers have been found to be consistent with the diagnosis of DLB, but their diagnostic specificity for the condition is not entirely clear. For this reason, they are not considered in the diagnosis of probable or possible DLB [20]. In most practices, and according to the American Academy of Neurology practice parameter for dementia [40], MRI or CT scans of the brain are routinely obtained in patients with suspected DLB. Certain findings on the structural neuroimaging can be supportive in the diagnosis. Unlike $\mathrm{AD}$ patients, DLB patients have been found to have minimal atrophy of their medial temporal lobes on brain imaging. This temporal lobe atrophy has been found to be more consistent with the tangle histopathology of $\mathrm{AD}$ than with the diffuse Lewy body histopathology of DLB [41]. SPECT and PET scans serve as additional supportive biomarkers for DLB when there are signs of generalized decreased perfusion/metabolism in patient brains, particularly when they are in the occipital region. SPECT perfusion measurement of cerebral blood flow using ${ }^{99 \mathrm{~m}} \mathrm{Tc}$-hexamethylpropyleneamine oxime (99mTc-HMPAO) has been shown to have $65 \%$ sensitivity and $87 \%$ specificity for DLB diagnosis against AD [42]. Brain imaging of metabolic function with glucose $\left({ }^{18} \mathrm{~F}-\mathrm{FDG}\right)$ PET scanning has been shown to be superior to $99 \mathrm{mTc}$-HMPAO perfusion scanning for accurate distinction between DLB and $\mathrm{AD}$, with a sensitivity of $70 \%$ and a specificity of $74 \%$ [43]. Despite their utility in distinguishing between AD and DLB in research, these tests do not have proven accuracy at the levels necessary to be considered as indicative biomarkers in the new diagnostic criteria for DLB [20]. EEG is not routinely used in the diagnosis of dementia, but has displayed utility in differentiation between DLB and AD when practitioners need additional data to distinguish between the two clinically. EEG in DLB patients has shown theta/delta waves in posterior and anterior/temporal deviations, as opposed to a majority of AD patients showing alpha waves in posterior deviations [44]. Genetics and fluid studies do not currently have any established utility in the diagnosis of DLB. Genome-wide association studies of patients who suffer from DLB, utilizing genotyping arrays, showed associations among the APOE, SNCA, GBA, BCL7C/ $S T X 1 B$, and GABRB3 loci and a positive diagnosis of DLB. Interestingly, the APOE locus has previously been shown to be associated with diagnoses of DLB and AD. This same study estimated the heritability of a diagnosis of DLB to be about $36 \%$ [45]. CSF studies may be helpful in distinguishing between DLB and other forms of dementia. The CSF of patients with DLB, like patients with AD, often contains increased amounts of tau protein. Higher concentrations of CSF tau have been correlated with earlier mortality in DLB patients [6]. Measurements of $A \beta$ peptide in CSF has been shown to be different in patients suffering from $\alpha$ synucleinopathies from control patients. One such study showed that CSF levels of A $1-42$ in DLB patients were decreased compared with patients who served as non-demented controls. This is similar to what has been seen in comparisons between $\mathrm{AD}$ patients and non-demented controls [46].

\section{TREATMENT/MANAGEMENT}

No cure or disease-modifying treatment for dementia with Lewy bodies exists. Treatment of dementia with Lewy bodies is complex and requires a team of multidisciplinary caregivers. The goal of treatment is to relieve the burdens posed by the many symptoms of the disease in addition to educating patients and caregivers about what to expect during the course of the disease. The pharmacologic interventions used for treatment of the disease potentially slow progression but do not stop it. Care of DLB patients requires using appropriate interventions (pharmacologic and non-pharmacologic) 
that are life-long and require adjustment to maintain optimal care throughout disease progression. Although there are no medications currently FDA approved for the treatment of dementia with Lewy bodies, medications currently used in the treatment of DLB have significant overlap with those that are FDA approved for the treatment of Alzheimer's disease. When considering the use of medication in DLB treatment, the medical benefits must be weighed against possible adverse effects from the use of these medications.

\section{Cholinesterase Inhibitors}

Cholinesterase inhibitors are one of the main pharmacologic treatments for dementia with Lewy bodies. They have been extensively studied for the treatment of Alzheimer's disease. The function of these pharmacologic agents is to increase the levels of acetylcholine within the brain, as acetylcholine is crucial to the maintenance of cognitive functions. Choline acetyltransferase, an important enzyme in the synthesis of acetylcholine, has been found to be more deficient in patients with DLB than in patients with Alzheimer's disease [47]. This cholinergic deficiency is thought to contribute to the neuropsychiatric symptoms of DLB. Cholinesterase inhibitors have shown efficacy in the improvement of cognitive functions of patients diagnosed with DLB. Many cholinesterase inhibitors are available for use in DLB; these include donepezil, rivastigmine, and galantamine. A comparative analysis of the three showed global improvement of cognitive and neuropsychiatric measures in DLB patients with their use; none of the three were found to have greater efficacy than the others [48]. Donepezil appears to be the most well studied and widely used of the cholinesterase inhibitors in the treatment of DLB. It was approved in 2014 for the treatment of DLB in Japan [49]. In randomized controlled trials, when compared with placebo, donepezil has been shown to improve mental status [50] and overall behavioral, cognitive, and global function in patients with DLB $[51,52]$. In patients where donepezil is well tolerated at a lower dose without adverse effects, increasing the dose from 5 to $10 \mathrm{mg} /$ day can improve cognitive function further [51. Multicenter open-label studies have proven the long-term safety of donepezil for the treatment of psychiatric and cognitive function [53]. One review of the available literature has shown that the use of donepezil in patients with DLB is effective in the treatment of neuropsychiatric symptoms [54]. Donepezil has been shown be relatively safe to use in patients with DLB without exacerbating existing extrapyramidal symptoms in long-term use [55]. Rivastigmine, another acetylcholinesterase inhibitor used in DLB, has shown efficacy in randomized controlled trials for treatment of DLB compared with placebo [56]. It is FDA approved for the treatment of mild-to-moderate Parkinson's disease dementia, which by definition is a type of Lewy body dementia [57]. Galantamine, a third acetylcholinesterase inhibitor used in DLB, has been investigated extensively for the treatment of Alzheimer's disease. However, no randomized controlled trials have been completed for galantamine in the treatment of DLB. An openlabel investigation of galantamine showed significant improvement in cognitive deficits and visual hallucinations in DLB patients who were treated with the medication after 24 weeks [58]. In the authors' clinical experience, treatment of DLB with cholinesterase inhibitors leads to a more profound clinical response than treatment of $\mathrm{AD}$ patients. Observed responses include decreased hallucination, decreased fluctuations, and increased alertness. With donepezil, doses $>$ $10 \mathrm{mg}$ (often $10 \mathrm{mg}$ in the morning after a meal and $5 \mathrm{mg}$ after the evening meal) are considered if tolerated because of the clinical response.

\section{Memantine}

Memantine, a glutamatergic NMDA receptor antagonist, may be useful in the treatment of DLB. However, research on the therapeutic effects of memantine is limited. Randomized controlled studies have shown moderate clinical improvement in the use of memantine in DLB versus placebo [59, 60]. A small-scale prospective randomized controlled trial has shown that compared with placebo memantine 
can improve the survival of DLB patients if initiated early on in the course of the disease [61]. In the authors' practice, it is not routinely used because of the lack of substantial clinical evidence, but can be considered.

\section{Levodopa}

The parkinsonism symptoms that may be present in patients with DLB can be managed similarly to the symptoms of idiopathic Parkinson's disease. DLB patients who suffer from parkinsonian movement symptoms can be treated with levodopa. Levodopa is preferred to dopamine agonists for the treatment of symptoms of parkinsonism in DLB. Dopamine agonists have been found to have greater potential for worsening neuropsychiatric symptoms such as hallucinations, confusion, and psychosis with less benefit in motor control than levodopa [27]. However, the responsiveness of these motor symptoms to levodopa is much less than the motor symptoms in patients with Parkinson's disease or Parkinson's disease dementia [62]. Extended follow-up of patients with DLB compared with $\mathrm{AD}$ who are treated with levodopa has yielded the same results. This shows that chronic long-term use of levodopa in DLB patients can yield a positive response in their motor symptoms, but not to the same extent as their peers with $\mathrm{AD}$ [63]. In the clinical practice of the authors, low doses of carbidopa/levodopa are often well tolerated, but moderate and high doses can precipitate worsening of hallucinations and should be used with caution.

\section{Neuroleptics}

Neuroleptics must be used with great caution in dementia with Lewy bodies. As stated previously, patients who suffer from DLB are known to have a very high sensitivity to the side effects of these medications [27]. Neuroleptics can exacerbate many of the symptoms associated with DLB, including cognitive fluctuations, motor symptoms, and dysautonomia. First generation antipsychotics should be avoided entirely in all patients with DLB. The authors often list a drug "allergy" to haloperidol in electronic medical records of patients who are diagnosed with DLB to further disincentivize the use of these drugs if a patient is hospitalized. If treatment of disturbing hallucinations or delusions is deemed necessary based on a major impact on patient quality of life, priority should be considered for quetiapine, which has the lowest incidence of extrapyramidal symptoms. A case series provided limited evidence for the use of quetiapine in DLB patients. Five of nine patients were found to have a positive response in a reduction of their psychotic symptoms and agitated behavior when treated with quetiapine [64]. The atypical antipsychotic olanzapine has been studied in patients with DLB. Randomized controlled trials of olanzapine in patients with DLB have produced mixed results. One RCT showed that the medication had little utility in the treatment of DLB [65], while another RCT showed that olanzapine was capable of reducing psychosis in DLB patients with worsening of their parkinsonian symptoms [66].

\section{Other Treatments}

REM sleep behavior disorder can be treated if this impacts patient or caregiver quality of life. The first-line therapy is typically to use melatonin at bedtime. Doses up to $15 \mathrm{mg}$ may be required. If melatonin is unsuccessful, clonazepam can be used to treat severe REM sleep behavior disorder. Hypersomnolence associated with DLB was been shown to be responsive to pharmacotherapy in a small study. A 12-week open-label pilot study of the psychostimulants modafinil and armodafinil has yielded some positive results. Improvement of several assessments related to wakefulness was observed with these therapeutic agents. Caregiver quality of life was shown to improve as well [67]. Nonpharmacologic interventions have been shown to have benefit in the management of patients who suffer from DLB. A systematic review of the literature on non-pharmacologic interventions found particular benefit in exercise therapy, physical therapy, patient/caregiver education, music therapy, occupational therapy, and interventions to prevent aspiration in patients with dysphagia [68]. 


\section{Future Pharmacologics and Clinical Trials}

Clinical trials specifically for the treatment of DLB have only begun in the USA in the last 2 years. The first interventional clinical trials for DLB were started by Axovant Sciences Corp. They began by developing two novel therapeutics known as intepirdine and nelotanserin. Intepirdine is a selective $5-\mathrm{HT}_{6}$ receptor antagonist. The HEADWAY-DLB Study was a doubleblind, placebo-controlled study to assess intepirdine's efficacy in reducing parkonsonian symptoms and improving cognitive symptoms as well as caregiver's interpretation of change and occurrence of adverse events evaluated by investigators. An additional trial to assess intepirdine's effect on gait was also completed. Unfortunately, preliminary results made recently available have shown that intepirdine made no significant difference compared with placebo for all study endpoints. While intepirdine was generally well tolerated in these studies, neither $35 \mathrm{mg}$ nor $70 \mathrm{mg}$ of intepirdine was found to make a significant difference in motor function, cognition, global function, or gait impairment [69-71].

Nelotanserin is a second novel therapeutic undergoing clinical trials as a treatment for DLB. Nelotanserin is an inverse agonist of serotonin receptors of the subtype $5-\mathrm{HT}_{2 \mathrm{~A}}$. Randomized clinical trials are ongoing to assess nelotanserin's safety and tolerance based on the incidence of adverse effects. These ongoing randomized-clinical trials are also observing changes in extrapyramidal symptoms and frequency of visual hallucinations; an open-label study is ongoing as well. Results are not yet available from these studies but prespecified analyses of the DLB patient subset have shown improvement in parkinsonian symptoms [a 4.00-point improvement in the Unified Parkinson's Disease Rating Schedule $(p=0.041$, unadjusted)] when nelotanserin was compared with placebo. In patients with a baseline Scale for the Assessment of Positive Symptoms > 8.0, nelotanserin treatment at $40 \mathrm{mg}$ for 2 weeks followed by $80 \mathrm{mg}$ for 2 weeks led to a 1.21 point improvement ( $p=0.011$, unadjusted). However, this improvement was not observed in the entire evaluable population. Nelotanserin was also generally well tolerated in the patients involved [72-74].

Several other companies have begun interventional clinical trials for DLB. Eli Lilly and Co. are openly recruiting for the Presence Study for patients with Parkinson's disease dementia and DLB [75]. This phase 2 study will be using a dopamine D1 receptor potentiator to look at a cognitive primary end point. Eisai, a Japanese pharmaceutical company and the maker of donepezil (Aricept), plans to open a phase 2 study for its drug E2027, a selective phosphodiesterase-9 inhibitor, in DLB patients [76]. This study will also pursue a cognitive end point.

\section{Stem Cell Therapy}

Stem cell therapy has been hypothesized as a treatment modality for DLB. Researchers developed an immune-deficient transgenic DLB murine model that exhibited progressive accumulation of human alpha-synuclein inclusions as well as cognitive and motor impairments, similar to that of patients suffering from DLB. Following grafts of human neural progenitor cells (CNS10-hNPCs), behavior assessments of the experimental murine model were completed at 30 and 90 days. Transplantation of these cells was found to rescue motor and cognitive deficits among the experimental murine model. Restoration of dopaminergic and glutamatergic regulation was additionally observed [77]. Although these animal study results are promising, clinical translation of these human neural progenitor cells may be complicated by the fact that immunosuppressive medications such as tacrolimus have been shown to modulate alpha-synuclein pathology [78].

\section{CONCLUSIONS}

While a great deal of progress has been made in the accurate diagnosis and effective treatment of DLB, important developments are still ongoing. Translational research activities developing new treatments for DLB are ongoing, and it will take time to understand the clinical impact. Raising awareness about DLB among the general population and physicians 
continues to be an important goal among advocates of DLB patients. Diagnosis of the disease is primarily clinical and requires primary care, specialty physicians and caregivers to remain vigilant for the early manifestations of DLB. It is the responsibility of the medical community to make the resources necessary for disease management available to patients and caregivers when faced with a diagnosis as daunting as DLB.

\section{ACKNOWLEDGEMENTS}

Funding. No funding or sponsorship was received for this study or publication of this article.

Authorship. All named authors meet the International Committee of Medical Journal Editors (ICMJE) criteria for authorship for this manuscript, take responsibility for the integrity of the work as a whole, and have given final approval to the version to be published.

Disclosures. Samuel Capouch has nothing to disclose. Martin Farlow has received grant/ research support from AbbVie, Accera, Axovant, Avanir, Biogen, Boehringer Ingelheim, Dominantly Inherited Alzheimer Network, Eisai, Eli Lilly, Genentech, Lundbeck, Novartis, Roche, and Suven Life Sciences, Ltd. He is part of the Consultant/Advisory Boards/DSMB Boards for Accera, Avanir, Axovant, AZTherapies, Cognition Therapeutics, Eli Lilly \& Co., FORUM Pharmaceuticals Inc. Research, KCRN Research, Kyowa Kirin Pharma, Longeveron, Medavante, Merck and Co. Inc., Proclara (formerly Neurophage Pharmaceuticals), Neurotrope Biosciences, Stemedica Cell Technologies Inc., Takeda, United Neuroscience Inc., and vTv Therapeutics. He is also affiliated with Elan. Jared R. Brosch has received grant/research support from AbbVie, Accera, Axovant, Avanir, Biogen, Boehringer Ingelheim, Dominantly Inherited Alzheimer Network, Eisai, Eli Lilly, Genentech, Lundbeck, Novartis, Roche, and Suven Life Sciences, Ltd.
Compliance with Ethics Guidelines. This article is based on previously conducted studies and does not contain any studies with human participants or animals performed by any of the authors.

Open Access. This article is distributed under the terms of the Creative Commons Attribution-NonCommercial 4.0 International License (http://creativecommons.org/licenses/ by-nc/4.0/), which permits any noncommercial use, distribution, and reproduction in any medium, provided you give appropriate credit to the original author(s) and the source, provide a link to the Creative Commons license, and indicate if changes were made.

\section{REFERENCES}

1. Spillantini MG, Schmidt ML, Lee VM, Trojanowski JQ, Jakes R, Goedert M. Alpha-synuclein in Lewy bodies. Nature. 1997;388(6645):839-40.

2. Plassman BL, Langa KM, Fisher GG, Heeringa SG, Weir DR, Ofstedal MB, et al. Prevalence of dementia in the United States: the aging, demographics, and memory study. Neuroepidemiology. 2007;29(1-2):125-32.

3. Vann Jones SA, O'Brien JT. The prevalence and incidence of dementia with Lewy bodies: a systematic review of population and clinical studies. Psychol Med. 2014;44(4):673-83.

4. McKeith IG, Galasko D, Kosaka K, Perry EK, Dickson DW, Hansen LA, et al. Consensus guidelines for the clinical and pathologic diagnosis of dementia with Lewy bodies (DLB): report of the consortium on DLB international workshop. Neurology. 1996;47(5):1113-24.

5. Savica R, Grossardt BR, Bower JH, Boeve BF, Ahlskog $\mathrm{JE}$, Rocca WA. Incidence of dementia with Lewy bodies and Parkinson disease dementia. JAMA Neurol. 2013;70(11):1396-402.

6. Mayo MC, Bordelon Y. Dementia with Lewy bodies. Semin Neurol. 2014;34(2):182-8.

7. Breitve $\mathrm{MH}$, Chwiszczuk LJ, Hynninen MJ, Rongve A, Bronnick K, Janvin C, et al. A systematic review of cognitive decline in dementia with Lewy bodies versus Alzheimer's disease. Alzheimers Res Ther. 2014;6(5-8):53. 
8. Walker Z, Allen RL, Shergill S, Mullan E, Katona CL. Three years survival in patients with a clinical diagnosis of dementia with Lewy bodies. Int J Geriatr Psychiatry. 2000;15(3):267-73.

9. Zweig YR, Galvin JE. Lewy body dementia: the impact on patients and caregivers. Alzheimers Res Ther. 2014;6(2):21.

10. Ku LJ, Pai MC, Shih PY. Economic impact of dementia by disease severity: exploring the relationship between stage of dementia and cost of care in Taiwan. PLoS One. 2016;11(2):e0148779.

11. Galvin JE, Duda JE, Kaufer DI, Lippa CF, Taylor A, Zarit SH. Lewy body dementia: caregiver burden and unmet needs. Alzheimer Dis Assoc Disord. 2010;24(2):177-81.

12. Williams MM, Xiong C, Morris JC, Galvin JE. Survival and mortality differences between dementia with Lewy bodies vs Alzheimer disease. Neurology. 2006;67(11):1935-41.

13. Bostrom F, Jonsson L, Minthon L, Londos E. Patients with dementia with lewy bodies have more impaired quality of life than patients with Alzheimer disease. Alzheimer Dis Assoc Disord. 2007;21(2):150-4.

14. Galvin JE, Duda JE, Kaufer DI, Lippa CF, Taylor A, Zarit SH. Lewy body dementia: the caregiver experience of clinical care. Parkinsonism Relat Disord. 2010;16(6):388-92.

15. Toledo JB, Cairns NJ, Da X, Chen K, Carter D, Fleisher A, et al. Clinical and multimodal biomarker correlates of ADNI neuropathological findings. Acta Neuropathol Commun. 2013;1:65.

16. Tsuboi Y, Uchikado H, Dickson DW. Neuropathology of Parkinson's disease dementia and dementia with Lewy bodies with reference to striatal pathology. Parkinsonism Relat Disord. 2007;13(Suppl 3):S221-4.

17. Mrak RE, Griffin WS. Dementia with Lewy bodies: definition, diagnosis, and pathogenic relationship to Alzheimer's disease. Neuropsychiatr Dis Treat. 2007;3(5):619-25.

18. Hamilton RL. Lewy bodies in Alzheimer's disease: a neuropathological review of 145 cases using alphasynuclein immunohistochemistry. Brain Pathol. 2000;10(3):378-84.

19. Muller T. Editorial regarding: practical treatment of Lewy body disease in the clinic: patient and physician perspectives. Neurol Ther. 2018;7(1):1-3.

20. McKeith IG, Boeve BF, Dickson DW, Halliday G, Taylor JP, Weintraub D, et al. Diagnosis and management of dementia with Lewy bodies: fourth consensus report of the DLB Consortium. Neurology. 2017;89(1):88-100.

21. Aarsland D, Kurz M. The eidemiology of dementia associated with Parkinson disease. J Neurol Sci. 2010;289(1-2):18-22.

22. Ferman TJ, Smith GE, Boeve BF, Graff-Radford NR, Lucas JA, Knopman DS, et al. Neuropsychological differentiation of dementia with Lewy bodies from normal aging and Alzheimer's disease. Clin Neuropsychol. 2006;20(4):623-36.

23. Bradshaw J, Saling M, Hopwood M, Anderson V, Brodtmann A. Fluctuating cognition in dementia with Lewy bodies and Alzheimer's disease is qualitatively distinct. J Neurol Neurosurg Psychiatry. 2004;75(3):382-7.

24. Tiraboschi P, Salmon DP, Hansen LA, Hofstetter RC, Thal LJ, Corey-Bloom J. What best differentiates Lewy body from Alzheimer's disease in early-stage dementia? Brain. 2006;129(Pt 3):729-35.

25. Hamilton JM, Landy KM, Salmon DP, Hansen LA, Masliah E, Galasko D. Early visuospatial deficits predict the occurrence of visual hallucinations in autopsy-confirmed dementia with Lewy bodies. Am J Geriatr Psychiatry. 2012;20(9):773-81.

26. Onofrj M, Taylor JP, Monaco D, Franciotti R, Anzellotti F, Bonanni L, et al. Visual hallucinations in PD and Lewy body dementias: old and new hypotheses. Behav Neurol. 2013;27(4):479-93.

27. McKeith I, Fairbairn A, Perry R, Thompson P, Perry E. Neuroleptic sensitivity in patients with senile dementia of Lewy body type. BMJ. 1992;305(6855):673-8.

28. Onofrj M, Varanese S, Bonanni L, Taylor J-P, Antonini A, Valente E, et al. Cohort study of prevalence and phenomenology of tremor in dementia with Lewy bodies. J Neurol. 2013;260:1731-42.

29. Aarsland D, Ballard C, McKeith I, Perry RH, Larsen JP. Comparison of extrapyramidal signs in dementia with Lewy bodies and Parkinson's disease. J Neuropsychiatry Clin Neurosci. 2001;13(3):374-9.

30. Boeve BF, Silber MH, Saper CB, Ferman TJ, Dickson DW, Parisi JE, et al. Pathophysiology of REM sleep behaviour disorder and relevance to neurodegenerative disease. Brain. 2007;130(Pt 11):2770-88.

31. Ferman TJ, Boeve BF, Smith GE, Silber MH, Kokmen E, Petersen RC, et al. REM sleep behavior disorder and dementia: cognitive differences when compared with AD. Neurology. 1999;52(5):951-7. 
32. Ferman TJ, Boeve BF, Smith GE, Silber MH, Lucas JA, Graff-Radford NR, et al. Dementia with Lewy bodies may present as dementia and REM sleep behavior disorder without parkinsonism or hallucinations. J Int Neuropsychol Soc. 2002;8(7):907-14.

33. Genier Marchand D, Montplaisir J, Postuma RB, Rahayel S, Gagnon JF. Detecting the cognitive prodrome of dementia with Lewy bodies: a prospective study of rem sleep behavior disorder. Sleep. 2017;40(1):1-11.

34. Dugger BN, Boeve BF, Murray ME, Parisi JE, Fujishiro H, Dickson DW, et al. Rapid eye movement sleep behavior disorder and subtypes in autopsy-confirmed dementia with Lewy bodies. Mov Disord. 2012;27(1):72-8.

35. Stubendorff K, Aarsland D, Minthon L, Londos E. The impact of autonomic dysfunction on survival in patients with dementia with Lewy bodies and Parkinson's disease with dementia. PLoS One. 2012;7(10):e45451.

36. McKeith I, O'Brien J, Walker Z, Tatsch K, Booij J, Darcourt J, et al. Sensitivity and specificity of dopamine transporter imaging with 123I-FP-CIT SPECT in dementia with Lewy bodies: a phase III, multicentre study. Lancet Neurol. 2007;6(4):305-13.

37. Chung EJ, Kim SJ. (123)I-Metaiodobenzylguanidine Myocardial Scintigraphy in Lewy body-related disorders: a literature review. J Mov Disord. 2015;8(2):55-66.

38. Oda H, Ishii K, Terashima A, Shimada K, Yamane Y, Kawasaki R, et al. Myocardial scintigraphy may predict the conversion to probable dementia with Lewy bodies. Neurology. 2013;81(20):1741-5.

39. Pao WC, Boeve BF, Ferman TJ, Lin SC, Smith GE, Knopman DS, et al. Polysomnographic findings in dementia with Lewy bodies. Neurologist. 2013;19(1):1-6.

40. Knopman D, DeKosky S, Cummings J, Chui H, Corey-Bloom J, Relkin N, et al. Practice parameter: diagnosis of dementia (an evidence-based review). Report of the quality standards subcommittee of the American Academy of Neurology. Neurology. 2001;56(9):1143-53.

41. Burton EJ, Barber R, Mukaetova-Ladinska EB, Robson J, Perry RH, Jaros E, et al. Medial temporal lobe atrophy on MRI differentiates Alzheimer's disease from dementia with Lewy bodies and vascular cognitive impairment: a prospective study with pathological verification of diagnosis. Brain. 2009;132(Pt 1):195-203.
42. Lobotesis K, Fenwick JD, Phipps A, Ryman A, Swann A, Ballard C, et al. Occipital hypoperfusion on SPECT in dementia with Lewy bodies but not AD. Neurology. 2001;56(5):643-9.

43. O'Brien JT, Firbank MJ, Davison C, Barnett N, Bamford C, Donaldson C, et al. 18F-FDG PET and perfusion SPECT in the diagnosis of Alzheimer and Lewy body dementias. J Nucl Med. 2014;55(12):1959-65.

44. Bonanni L, Thomas A, Tiraboschi P, Perfetti B, Varanese S, Onofrj M. EEG comparisons in early Alzheimer's disease, dementia with Lewy bodies and Parkinson's disease with dementia patients with a 2-year follow-up. Brain. 2008;131(Pt 3):690-705.

45. Guerreiro R, Ross OA, Kun-Rodrigues C, Hernandez DG, Orme T, Eicher JD, et al. Investigating the genetic architecture of dementia with Lewy bodies: a two-stage genome-wide association study. Lancet Neurol. 2018;17(1):64-74.

46. Kanemaru K, Kameda N, Yamanouchi H. Decreased CSF amyloid beta42 and normal tau levels in dementia with Lewy bodies. Neurology. 2000;54(9):1875-6.

47. Tiraboschi P, Hansen LA, Alford M, Sabbagh MN, Schoos B, Masliah E, et al. Cholinergic dysfunction in diseases with Lewy bodies. Neurology. 2000;54(2):407-11.

48. Bhasin M, Rowan E, Edwards K, McKeith I. Cholinesterase inhibitors in dementia with Lewy bodies: a comparative analysis. Int J Geriatr Psychiatry. 2007;22(9):890-5.

49. Inc. E. ARICEPT ${ }^{\circledR}$ approved in Japan as treatment for dementia with Lewy bodies. 2014. https://www. eisai.com/news/news201452.html. Accessed 01 June 2018.

50. Ikeda M, Mori E, Matsuo K, Nakagawa M, Kosaka K. Donepezil for dementia with Lewy bodies: a randomized, placebo-controlled, confirmatory phase III trial. Alzheimers Res Ther. 2015;7(1):4.

51. Mori E, Ikeda M, Nakai K, Miyagishi H, Nakagawa $\mathrm{M}$, Kosaka K. Increased plasma donepezil concentration improves cognitive function in patients with dementia with Lewy bodies: an exploratory pharmacokinetic/pharmacodynamic analysis in a phase 3 randomized controlled trial. J Neurol Sci. 2016;366:184-90.

52. Mori E, Ikeda M, Kosaka K. Donepezil DLBSI. Donepezil for dementia with Lewy bodies: a randomized, placebo-controlled trial. Ann Neurol. 2012;72(1):41-52. 
53. Ikeda M, Mori E, Kosaka K, Iseki E, Hashimoto M, Matsukawa N, et al. Long-term safety and efficacy of donepezil in patients with dementia with Lewy bodies: results from a 52-week, open-label, multicenter extension study. Dement Geriatr Cogn Disord. 2013;36(3-4):229-41.

54. Cummings J, Lai TJ, Hemrungrojn S, Mohandas E, Yun Kim S, Nair G, et al. Role of donepezil in the management of neuropsychiatric symptoms in Alzheimer's disease and dementia with Lewy bodies. CNS Neurosci Ther. 2016;22(3):159-66.

55. Mori E, Ikeda M, Nakagawa M, Miyagishi H, Yamaguchi H, Kosaka K. Effects of donepezil on extrapyramidal symptoms in patients with dementia with Lewy bodies: a secondary pooled analysis of two randomized-controlled and two open-label long-term extension studies. Dement Geriatr Cogn Disord. 2015;40(3-4):186-98.

56. McKeith I, Del Ser T, Spano P, Emre M, Wesnes K, Anand R, et al. Efficacy of rivastigmine in dementia with Lewy bodies: a randomised, double-blind, placebo-controlled international study. Lancet. 2000;356(9247):2031-6.

57. FDA. Exelon prescribing information. 2015. https:// www.accessdata.fda.gov/drugsatfda_docs/label/ 2015/020823s032,021025s022lbl.pdf. Accessed 01 June 2018.

58. Edwards K, Royall D, Hershey L, Lichter D, Hake A, Farlow M, et al. Efficacy and safety of galantamine in patients with dementia with Lewy bodies: a 24-week open-label study. Dement Geriatr Cogn Disord. 2007;23(6):401-5.

59. Wesnes KA, Aarsland D, Ballard C, Londos E. Memantine improves attention and episodic memory in Parkinson's disease dementia and dementia with Lewy bodies. Int J Geriatr Psychiatry. 2015;30(1):46-54.

60. Aarsland D, Ballard C, Walker Z, Bostrom F, Alves G, Kossakowski $\mathrm{K}$, et al. Memantine in patients with Parkinson's disease dementia or dementia with Lewy bodies: a double-blind, placebo-controlled, multicentre trial. Lancet Neurol. 2009;8(7):613-8.

61. Stubendorff K, Larsson V, Ballard C, Minthon L, Aarsland D, Londos E. Treatment effect of memantine on survival in dementia with Lewy bodies and Parkinson's disease with dementia: a prospective study. BMJ Open. 2014;4(7):e005158.

62. Molloy S, McKeith IG, O'Brien JT, Burn DJ. The role of levodopa in the management of dementia with Lewy bodies. J Neurol Neurosurg Psychiatry. 2005;76(9):1200-3.
63. Lucetti C, Logi C, Del Dotto P, Berti C, Ceravolo R, Baldacci $F$, et al. Levodopa response in dementia with lewy bodies: a 1-year follow-up study. Parkinsonism Relat Disord. 2010;16(8):522-6.

64. Takahashi H, Yoshida K, Sugita T, Higuchi H, Shimizu T. Quetiapine treatment of psychotic symptoms and aggressive behavior in patients with dementia with Lewy bodies: a case series. Prog Neuropsychopharmacol Biol Psychiatry. 2003;27(3):549-53.

65. Walker Z, Grace J, Overshot R, Satarasinghe S, Swan A, Katona CL, et al. Olanzapine in dementia with Lewy bodies: a clinical study. Int J Geriatr Psychiatry. 1999;14(6):459-66.

66. Cummings JL, Street J, Masterman D, Clark WS. Efficacy of olanzapine in the treatment of psychosis in dementia with lewy bodies. Dement Geriatr Cogn Disord. 2002;13(2):67-73.

67. Lapid MI, Kuntz KM, Mason SS, Aakre JA, Lundt ES, Kremers W, et al. Efficacy, safety, and tolerability of armodafinil therapy for hypersomnia associated with dementia with Lewy bodies: a pilot study. Dement Geriatr Cogn Disord. 2017;43(5-6):269-80.

68. Connors M, Quinto L, McKeith I, Brodaty H, Allan L, Bamford C, Thomas A, Taylor J-P, O'Brien J. Nonpharmacological interventions for Lewy body dementia: a systematic review. Psychol Med. 2017. https://doi.org/10.1017/S0033291717003257.

69. Study evaluating intepirdine (RVT-101) on gait and balance in subjects with dementia. https:// ClinicalTrials.gov/show/NCT02910102. Accessed 01 June 2018.

70. Study evaluating intepirdine (RVT-101) in subjects with dementia with lewy bodies: the HEADWAYDLB Study. https://ClinicalTrials.gov/show/ NCT02669433. Accessed 01 June 2018.

71. Long-Term Extension Study of Intepirdine (RVT101) in subjects with dementia with lewy bodies: HEADWAY-DLB Extension. https://ClinicalTrials. gov/show/NCT02928445. Accessed 01 June 2018.

72. Open-label study of nelotanserin in lewy body dementia with visual hallucinations or rem sleep behavior disorder. https://ClinicalTrials.gov/show/ NCT02871427. Accessed 01 June 2018.

73. Study evaluating nelotanserin for treatment of visual hallucinations in subjects with lewy body dementia. https://ClinicalTrials.gov/show/ NCT02640729. Accessed 01 June 2018.

74. Study evaluating nelotanserin for treatment of rem sleep behavior disorder in subjects with dementia 
(DLB or PDD). https://ClinicalTrials.gov/show/ NCT02708186. Accessed 01 June 2018.

75. Company ELa. A study of LY3154207 in participants with parkinson's disease dementia (PRESENCE). https://www.clinicaltrials.gov/ct2/show/ NCT03305809. Accessed 01 June 2018.

76. Eisai Inc. study to evaluate the efficacy, safety and tolerability of e2027 in subjects with dementia with lewy bodies. https://www.clinicaltrials.gov/ct2/ show/NCT03467152. Accessed 01 June 2018.
77. Goldberg NRS, Marsh SE, Ochaba J, Shelley BC, Davtyan H, Thompson LM, et al. Human neural progenitor transplantation rescues behavior and reduces alpha-synuclein in a transgenic model of dementia with lewy bodies. Stem Cells Transl Med. 2017;6(6):1477-90.

78. Gerard M, Debyser Z, Desender L, Baert J, Brandt I, Baekelandt V, et al. FK506 binding protein 12 differentially accelerates fibril formation of wild type alpha-synuclein and its clinical mutants A30P or A53T. J Neurochem. 2008;106(1):121-33. 